CLINICAL STUDY

\title{
TXNIP is highly regulated in bone biopsies from patients with endogenous Cushing's syndrome and related to bone turnover
}

\author{
Tove Lekva $^{1,2,4}$, Thor Ueland ${ }^{2,4}$, Hege Bøyum ${ }^{1,3}$, Johan Arild Evang ${ }^{1,2,4}$, Kristin Godang ${ }^{1}$ and Jens Bollerslev $^{1,4}$ \\ ${ }^{1}$ Section of Specialized Endocrinology, Department of Endocrinology, ${ }^{2}$ Research Institute for Internal Medicine, ${ }^{3}$ Division of Obstetrics and Gynaecology, \\ National Resource Centre for Women's Health and ${ }^{4}$ Faculty of Medicine, University of Oslo and Oslo University Hospital, O027 Oslo, Norway
}

(Correspondence should be addressed to T Lekva who is now at Section of Specialized Endocrinology and Institute for Internal Medicine, Oslo University Hospital, Rikshospitalet, University of Oslo, PO Box 4950 Nydalen, 0424 Oslo, Norway; Email: tove.lekva@medisin.uio.no)

\begin{abstract}
Objective: Patients with endogenous Cushing's Syndrome (CS), as long-time treated patients with exogenous glucocorticoids (GCs), have severe systemic manifestations including secondary osteoporosis and low-energy fractures. The aim of the present study was to investigate the functional role of TXNIP in bone with focus on osteoblast (OB) differentiation and OB-mediated osteoclast activity and function in vitro.

Design and methods: Nine bone biopsies from CS before and after surgical treatment were screened for expressional candidate genes. Microarray analyses revealed that the gene encoding TXNIP ranked among the most upregulated genes. Subsequent in vitro and in vivo studies were performed.

Results: We found that TXNIP gene in bone is downregulated in CS following surgical treatment. Furthermore, our in vivo data indicate novel associations between thioredoxin and TXNIP. Our in vitro studies showed that silencing TXNIP in OBs was followed by increased differentiation and expression and secretion of osteocalcin as well as enhanced activity of alkaline phosphatase. Moreover, treating osteoclasts with silenced TXNIP OB media showed an increased osteoclast activity.

Conclusions: TXNIP expression in bone is highly regulated during the treatment of active CS, and by GC in bone cells in vitro. Our data indicate that TXNIP may mediate some of the detrimental effects of GC on $\mathrm{OB}$ function as well as modulate OB-mediated osteoclastogenesis by regulating the OPG/RANKL ratio.
\end{abstract}

European Journal of Endocrinology 166 1039-1048

\section{Introduction}

Glucocorticoids (GCs) are widely used as antiinflammatory and immunosuppressive agents to control different acute and chronic diseases. Hypercortisolism is a well-known cause of bone loss, and patients with endogenous Cushing's syndrome (CS) frequently display low bone mass and fragility fractures. Cortisol excess inhibits bone formation, increases bone resorption, impairs calcium absorption from the gut, and affects the secretion of several hormones, cytokines, and growth factors with potential influence on bone metabolism (1). Sustained and prolonged hypercortisolism leads to a rapid early phase of bone mineral density (BMD) decline due to higher bone resorption, which is followed later by a slower and more progressive phase of impaired bone formation (2). The initial effect of GC at the cellular level is stimulation of osteoblastic (OB) cells to increase the production of RANKL and reduce the production of OPG (2), thereby turning the RANKL/ OPG ratio in favor of osteoclastogenesis $(3,4)$. Moreover, $\mathrm{GC}$ directly reduces the number and function of $\mathrm{OB}$, the latter as the most detrimental effect of GC on bone metabolism (2). At the bone resorptive site, GC acts directly on osteoclasts and prolongs their life span and thereby the resorptive capacity (2).

Thioredoxin 1 (TRX) regulates the redox state of cells and is induced and secreted in response to oxidative stress and inflammation. Elevation of serum/plasma TRX levels has been reported in patients with diabetes mellitus, impaired glucose tolerance, arterial hypertension, hypercholesterolemia, atherosclerosis $(5,6,7)$, and various types of malignancies (8). Several studies suggest that TRX activity can be modulated by the interaction with thioredoxin interacting protein-1 (TXNIP, also called VDUP-1 for vitamin D3-upregulated protein or TBP-2 for TRX-binding protein-2) $(9,10)$. TXNIP is a multifunctional protein and a major messenger of intracellular physiological processes and upregulated by various types of stress (9). Its expression has been shown to be induced by dexamethasaone (Dex) and may mediate GC-induced apoptosis (11).

In order to search for regulated genes in target tissues of potential pathogenetic importance, we used global gene expression profiling of bone biopsies from CS patients, before and 3 months after surgical treatment, 
to screen for changes in gene expression. We found the gene encoding TXNIP as one of the most regulated genes in relation to treatment. Based on the well-known effects of GC excess on bone tissue, we investigated whether TXNIP could modulate OB differentiation and osteocalcin (OC) synthesis in OB. Moreover, we investigated the effects of $\mathrm{OB}$ signaling on osteoclast activity and function in vitro.

\section{Materials and methods}

\section{Subjects}

Nine patients diagnosed with CS (12) were recruited based on clinical evaluation and biochemical workup (13, 14). Biological specimens (blood, bone biopsies) were first collected at baseline (untreated), and then again $3 \pm 1.3$ (mean \pm s.D.) months after surgical treatment. Twenty-three new CS patients were recruited in the period 2002-2009 (blood samples before and after treatment from 16 patients, $0.9 \pm 0.9$ (mean \pm s.D.) years after surgical treatments). Seventeen and eight of the female patients were pre- and postmenopausal respectively. Twenty-three patients were diagnosed with Cushing's disease (two of them removed adrenal cortex adenoma at a later time), eight with adrenal cortex adenoma, and one with an ectopic ACTH-producing adenoma. CS patients received tapering doses of cortisone in the postoperative phase. A group of 29 healthy subjects matched by age, sex, and body mass index were used as controls. Blood samples were drawn fasting in the morning. All patients and controls gave signed informed consent, and the study was approved by the Regional Committee of Medical and Health Research Ethics (REK) in Eastern Norway and conducted according to the Declaration of Helsinki II.

\section{Bone biopsies}

Transiliac crest bone biopsies with an inner diameter of $6 \mathrm{~mm}$ were obtained from CS patients under local anesthesia and stored in liquid nitrogen until analysis (15). Alternative hips were used for the two biopsies. All microarray raw data have been deposited on Gene Expression Omnibus (GEO) under accession number GSE30159.

\section{Biochemical markers}

Circulating OC was measured by EIA, provided by Quidel (Santa Clara, CA, USA) and N-terminal telopeptides of type-1 collagen (NTX), TRX by ELISA, Redox Bioscience, Inc. (Osaka, Japan), and cortisol was measured as previously reported (12). Cortisol measurement also detects cortisone acetate.

\section{BMD measurement}

BMD was measured by dual-energy X-ray absorptiometry (LUNAR Corp., Madison, WI, USA) (13). The values were expressed as Z-scores.

\section{RNA isolation from bone biopsies and cultured cells}

Extraction of total RNA was performed using Trizol (Invitrogen) as previously described (12). RNA was purified using Qiagen RNeasy micro kit (Qiagen). The integrity was assessed using Agilent 2100 Bioanalyzer (Agilent Technologies, Santa Clara, CA, USA), and concentrations determined by optical density (OD) readings on Nanodrop ND-1000 Spectrophotometer (Nanodrop Technologies, Wilmington, DE, USA).

\section{Microarray}

Microarray analysis was performed as previously described (12), using biotin-labeled cRNA probes hybridized to HG U133 Plus 2.0 Arrays (Affymetrix, Inc., Santa Clara, CA, USA).

\section{Real-time RT-PCR}

RT was performed using a High Capacity cDNA Archive kit (Applied Biosystems, Foster City, CA, USA). Quantification of mRNA was performed using standard curve method of the ABI Prism 7500 (Applied Biosystems). For real-time RT-PCR, sequence-specific oligonucleotide primers were designed using Primer Express Software version 2.0 (Applied Biosystems; Table 1).

\section{Cell culture}

The human fetal OB (hFOB) cell line 1.19 was obtained from American Type Culture Collection (CRL-11372, Rockville, MD, USA) $(16,17)$. hFOB cells proliferate and exhibit early $\mathrm{OB}$ characteristics at $33.5^{\circ} \mathrm{C}$ (the permissive temperature, where the temperature-sensitive mutant SV 40 large T antigen is active), but have the ability to acquire mature $\mathrm{OB}$ features when grown at $39.5{ }^{\circ} \mathrm{C}$ (the restrictive temperature, when the SV 40 large $\mathrm{T}$ antigen is inactive).

\section{Cellular differentiation and Dex treatment}

Cells were grown at $33.5{ }^{\circ} \mathrm{C}$ until $90 \%$ confluence before shifting the temperature to $39.5{ }^{\circ} \mathrm{C}$ to induce differentiation for 2 days or more, as indicated. For time-course and dose-response experiments hFOB cells were seeded in triplicate cultures (24-well plates; $3.0 \times$ $10^{4}$ cells/well) and differentiated at $39.5^{\circ} \mathrm{C}$ for 2 days, followed by treatment with Dex $\left(10^{-8}\right.$ and $\left.10^{-6} \mathrm{M}\right)$ or vehicle $(0.6 \%$ ethanol), as indicated. 
Table 1 Primer sequences used in PCR reactions.

\begin{tabular}{|c|c|c|c|c|}
\hline Genes & GenBank & Forward $\left(5^{\prime}-3^{\prime}\right)$ & Reverse $\left(5^{\prime}-3^{\prime}\right)$ & Size (bp) \\
\hline$A L P(A L P 1)$ & NM_000478 & GGAAGAGCTTCAAACCGAGA & TGGCTCGAAGAGACCCAATA & 109 \\
\hline RUNX2 & $\begin{array}{l}\text { NM_001024630 } \\
\text { NM_001015051 } \\
\text { NM_004348 }\end{array}$ & CGGAATGCCTCTGCTGTTAT & GGTGAAACTCTTGCCTCGTC & 89 \\
\hline$O C$ & NM 19917 & ССТСАСАСТССТСGСССТАТТ & GGACACAAAGGCTGCACCTT & 105 \\
\hline COL1A2 & NM_000089 & ATGCTGGCAGCCAGTTTGA & TGCAGTGGTAGGTGATGTTCTGA & 119 \\
\hline$\beta$-Actin & NM_001101 & AGGCACCAGGGCGTGAT & TCGTCCCAGTTGGTGACGAT & 127 \\
\hline RANKL & $\begin{array}{l}\text { NM_033012 } \\
\text { NM_003701 }\end{array}$ & GTGCAAAAGGAATTACAACATATCGT & AACCATGAGCCATCCACCAT & 76 \\
\hline$O P G$ & NM 002546 & АATCAACTCAAAAATGTGGAATAGATGT & GCGTAAACTTTGTAGGAACAGCAA & 80 \\
\hline TXNIP & NM_006472.3 & GAAGCTCCTCCCTGCTATATGGA & CCATGTCATCTAGCAGAGGAGTTGT & 81 \\
\hline Caspase 3 & $\begin{array}{l}\text { NM_004346 } \\
\text { NM_032991.2 }\end{array}$ & TGGATTATCCTGAGATGGGTTT & CAGACCGAGATGTCATTCCA & 80 \\
\hline Caspase 8 & $\begin{array}{l}\text { NM_001228 } \\
\text { NM_033358.3 }\end{array}$ & AGTGGGCAAGAGAATTAGCATTT & TCTCAATATTCCCAAGGTTCAAG & 138 \\
\hline$B C L 2$ & NM_000633 & CGGTGGTGGAGGAGCTCTT & TGACGCTCTCCACACACATGA & 93 \\
\hline$B A X$ & $\begin{array}{l}\text { NM_004324 } \\
\text { NM_138761.3 } \\
\text { NM_138763 } \\
\text { NM_138764 }\end{array}$ & CCGCCGTGGACACAGACT & CGGCCCCAGTTGAAGTTG & 84 \\
\hline
\end{tabular}

\section{Alkaline phosphatase staining}

Alkaline phosphatase (ALP) staining was determined using Sigma kit 104-LS, as previously described (Sigma) (18).

\section{Silencing of TXNIP gene expression in hFOB}

A pool of four siRNA duplexes specific for human TXNIP (silencing RNA TXNIP (siTXNIP)) and scramble control pool (silencing RNA scramble control (siSCR)) were designed by Dharmacon (Lafayette, CO, USA). The sense strand sequences were TXNIP1, 5'-GAAUACAUGUUCCCGAAUUUU-3'; TXNIP2, 5'-GCAAACAGACUUCGGAGUAUU-3'; TXNIP3, 5'-CGAACCAGCUCUGAGAUGAUU-3'; TXNIP4, 5'-GUCAGAGGCAAUCAUA UUAUU- $3^{\prime}$. hFOB cells were grown in 12-well plates $\left(5 \times 10^{4}\right.$ cells/well $)$ for $24 \mathrm{~h}$ at $33.5^{\circ} \mathrm{C}(40 \%$ confluent) in antibiotic-free medium containing $10 \%$ FCS. For transfection, $50 \mathrm{nM}$ siRNA duplexes and $6 \mu \mathrm{l}$ HiPerFect transfection reagent (Qiagen) were prepared in OptiMem with glutamax-1 (Gibco-Invitrogen) at a final volume of $300 \mu \mathrm{l}$ in 12-well plates (Costar, Cambridge, MA, USA). After $4 \mathrm{~h}, 300 \mu \mathrm{l}$ medium with 0.6\% Ultroser (Pall Corp., Port Washington, NY, USA) were added to the cells for the remaining period of $24 \mathrm{~h}$ at $33.5^{\circ} \mathrm{C}$, and the temperature was shifted to $39.5^{\circ} \mathrm{C}$ to induce differentiation.

\section{Silencing of TXNIP in normal human OBs}

The normal human OBs (NHOst) cell line was obtained from Lonza (Walkersville, Inc., Walkersville, MD, USA) and silenced for 2 days with siTXNIP and siSCR. For transfection, $50 \mathrm{nM}$ siRNA duplexes and $6 \mu \mathrm{l} \mathrm{HiPerFect}$ transfection reagent (Qiagen) were prepared in OptiMem with glutamax-1 (Gibco-Invitrogen) at a final volume of $300 \mu \mathrm{l}$ in 12-well plates (Costar,). After $4 \mathrm{~h}$, $300 \mu \mathrm{l}$ differentiation media recommended from the manufacturer were added to the cells for the remaining period of 2 and 7 days, changing the media after 4 days.

\section{OC secretion from hFOB}

OC measurement from supernatant of siTXNIP and siSCR hFOB and NHOst was performed using the Human Gla-Osteocalcin High Sensitive EIA kit provided by Takara (Shiga, Japan).

\section{Lactate dehydrogenase cytotoxicity assay}

A lactate dehydrogenase (LDH) cytotoxicity assay kit (Roche) was used for quantification of plasma membrane damage in hFOB differentiated for 2 days followed by treatment with Dex $\left(10^{-6} \mathrm{M}\right)$ or vehicle $(0.6 \%$ ethanol) for $24 \mathrm{~h}$. One hundred microliters of supernatant were transferred into corresponding wells of a clear 96-well plate and followed by addition of $100 \mu \mathrm{l}$ of the reaction mixture to each well and incubation for $30 \mathrm{~min}$ in the dark. The absorbance at $490 \mathrm{~nm}$ was measured using a microplate reader to analyze the release of $\mathrm{LDH}$ into the media.

\section{Western blot}

Cells were washed twice in PBS, scraped in lysis buffer, and stored at $-20{ }^{\circ} \mathrm{C}$ until use. Eighty micrograms of total protein of each extract were incubated at $97^{\circ} \mathrm{C}$ for 2 min before being separated on any kDs SDS-PAGE gels (Bio-Rad Laboratories) and transferred onto polyvinylidene difluoride membranes (NEN; Life Science, Boston, MA, USA). Membranes were incubated with mouse anti-human TXNIP (MBL International, Woburn, MA, USA) antibody over night at $4{ }^{\circ} \mathrm{C}$ and for $1 \mathrm{~h}$ at room 
temperature with anti-mouse IgG (Cell Signaling Technology, Denver, MA, USA) HRP-conjugated secondary antibody. HRP signals were developed using the ECL Plus western blotting detection system (GE Healthcare Bio-Sciences, Uppsala, Sweden) and visualized by Luminiscent Image Analyzer 4000 mini (Fujifilm, Tokyo, Japan). To ensure equal loading of protein the blots were stripped and reprobed with antibody against $\beta$-actin (Sigma-Aldrich).

\section{Differentiation and Dex treatment of osteoclasts}

Human osteoclast precursor cells (Lonza Walkersville, Inc.) were cultured for 8 days. At day 5, the cells were treated with different doses of Dex $\left(10^{-11}\right.$ to $\left.10^{-8} \mathrm{M}\right)$ or vehicle $(0.1 \%$ ethanol $)$ for $48 \mathrm{~h}$.

\section{Silencing of TXNIP in osteoclasts}

Human osteoclast precursors were grown in quadruplicate cultures in 12-well plates for 6 days. The same siRNA duplexes specific for human TXNIP were used as in the hFOB experiment. For transfection, $50 \mathrm{nM}$ siRNA duplexes and $6 \mu \mathrm{l}$ HiPerFect transfection reagent (Qiagen) were prepared in OptiMem with glutamax-1 (Gibco-Invitrogen) at a final volume of $300 \mu \mathrm{l}$. After $4 \mathrm{~h}, 300 \mu \mathrm{l}$ medium were added to the cells for the remaining period of 2 days, including cells treated with vehicle $(0.1 \%$ ethanol $)$ and Dex $\left(10^{-6} \mathrm{M}\right)$ for $24 \mathrm{~h}$.

\section{Osteoclasts stimulated with silenced TXNIP OB medium}

Human osteoclast precursors were grown in quadruplicate cultures in 12-well plates for 6 days before they were cultured 50/50 with normal growth medium and media from hFOB (siTXNIP and siSCR treated with and without Dex $\left(10^{-6} \mathrm{M}\right)$ or vehicle, for $24 \mathrm{~h}$ in the silencing experiment) for 2 days.

\section{Tartrate-resistance acid phosphatase activity in osteoclasts media}

Conditioned media and freshly prepared reaction buffer were added to a 96-well plate as previously described (12). The reaction was left in the dark $1 \mathrm{~h}$ at $37^{\circ} \mathrm{C}$ and stopped with $100 \mu \mathrm{l}$ of $0.3 \mathrm{M} \mathrm{NaOH}$ and $\mathrm{OD}$ was measured at $405 \mathrm{~nm}$.

\section{Osteoclasts from CD14 + monocytes}

CD14+ monocytes were selected using MACS cell sorting system (Miltenyi Biotech, Surrey, UK). Peripheral blood mononuclear cells (PBMCs) were isolated from heparinized venous blood obtained from healthy female donors, and diluted twofold in saline. PBMCs were obtained by centrifugation through Ficoll; $30 \mathrm{ml}$ of diluted blood were layered onto $10 \mathrm{ml}$ of lymphoprep solution (Nycomed, Birmingham, UK) in a sterile $50 \mathrm{ml}$ tube (Falcon, BD Biosciences, San Jose, CA, USA). Blood was then subjected to centrifugation at $350 \boldsymbol{g}$ for $25 \mathrm{~min}$. Cells at the interface were removed and washed twice in RPMI (Gibco-Invitrogen Corp.) and counted and seeded into 24 -well plates, $3 \times 10^{5}$ cells/well.

Osteoclasts were differentiated and maintained in $\alpha$-minimal essential medium (Gibco, Invitrogen Corp.) with $10 \%$ fetal bovine serum (FBS), L-glutamine $(2 \mathrm{mM})$, penicillin/streptomycin $(50 \mathrm{U} / \mathrm{ml}$ and $50 \mu \mathrm{g} / \mathrm{ml})$ in a humidified atmosphere $\left(37^{\circ} \mathrm{C}, 5 \%\right.$ $\mathrm{CO}_{2}$ ) containing macrophage colony-stimulating factor (M-CSF) and RANKL (25 ng/ml; R\&D, Minneapolis, MN, USA) changing media every 4-5 days. On day 16 , osteoclasts were treated with siTXNIP and siSCR or siTXNIP and siSCR OB media from the hFOB silencing experiment for 2 days in $\alpha$-minimal essential medium with $0.6 \%$ Ultroser.

\section{Osteoassay human bone plate}

$\mathrm{CD} 14+$ monocytes were seeded onto osteoassay human bone plate (Lonza Walkersville, Inc.), $5 \times 10^{4}$ cells/well. Osteoclasts were differentiated and maintained in $\alpha$-minimal essential medium (Gibco, Invitrogen Corp.) with 10\% FBS, L-glutamine $(2 \mathrm{mM})$, penicillin/streptomycin $(50 \mathrm{U} / \mathrm{ml}$ and $50 \mu \mathrm{g} / \mathrm{ml})$ in a humidified atmosphere $\left(37^{\circ} \mathrm{C}, 5 \% \mathrm{CO}_{2}\right)$ containing M-CSF and RANKL ( $25 \mathrm{ng} / \mathrm{ml}$; R\&D) changing media every 4-5 days. On day 16 , osteoclasts were treated with condition media from siTXNIP and siSCR OB and resorption media from the calcifluor kit (Lonza Walkersville, Inc.) with $0.1 \%$ FCS, (50/50) for 5 days on osteoassay plates. Calcium release using the Calcifluor assay kit was measured by fluorescence, directly proportional to the calcium content.

\section{Statistical analysis}

Unless stated otherwise, all values are expressed as mean \pm s.e.m. Wilcoxon ranked sum test was used to evaluate differences between the sample of interest and its own control in bone biopsies and serum samples. For analysis of time-course and doseresponse, one-way ANOVA was used. Unpaired Student's sample $t$-test (two-tailed) was used to evaluate differences between control and treated cells and siTXNIP and scramble control-treated cells. Between controls and CS samples nonparametric Mann-Whitney was used. Relationships between variables were tested by Spearman's correlation $(R)$. Average signal levels on the microarrays for all differentially regulated mRNAs included were at least 50 , with a $P$ value $<0.05$ (paired $t$-test). A $P$ value of $<0.05$ was considered significant. All in vitro 
Table 2 Baseline characteristics of the study population. Data are given as mean \pm s.D. BMD values are given as Z-scores.

\begin{tabular}{lccc}
\hline & Controls $(n=29)$ & $\begin{array}{c}\text { Cushing pre-surgery } \\
(n=32)\end{array}$ & $\begin{array}{c}\text { Cushing post-surgery } \\
(n=16)\end{array}$ \\
\hline Demographics & & & \\
Age (years) & $45 \pm 11$ & $41 \pm 11$ & $44 \pm 10$ \\
Women/men $(n)$ & $19 / 10$ & $25 / 7$ & $13 / 3$ \\
BMI $\left(\mathrm{kg} / \mathrm{m}^{2}\right)$ & $27.6 \pm 4.4$ & $30.3 \pm 7.0$ & $31.2 \pm 7.5$ \\
Cortisol (nmol/l) & $417 \pm 214.3$ & $634 \pm 214.6^{\mathrm{a}}$ & $163 \pm 174.2^{\mathrm{a}, \mathrm{b}}$ \\
Female pre/postmenopausal & $\mathrm{ND}$ & $17 / 8$ & $\mathrm{ND}$ \\
BMD & & & $\mathrm{ND}$ \\
Total & $0.53 \pm 1.1$ & $-0.89 \pm 1.3^{\mathrm{a}}$ & $\mathrm{ND}$ \\
Lumbar spine & $0.12 \pm 1.4$ & $-1.77 \pm 1.5^{\mathrm{a}}$ & $\mathrm{ND}$ \\
Femoral neck & $0.20 \pm 0.9$ & $-1.62 \pm 1.3^{\mathrm{a}}$ & $\mathrm{ND}$ \\
Radius ultradistal & $0.40 \pm 1.3$ & $-1.67 \pm 1.5^{\mathrm{a}}$ & \\
\hline
\end{tabular}

andicates significant levels between controls and CS

${ }^{\mathrm{b}}$ Between CS pre- and post-surgery.

studies were performed three times with representative experiments presented.

\section{Results}

\section{In vivo}

Patient's characteristics are given in Table 2. Patients with CS were characterized and compared with age, body mass index, and sex-matched healthy controls. Cortisol levels were increased compared with controls and decreased following surgery, reaching levels that were lower than the controls. BMD in CS patients were significantly lower compared with controls for all compartments investigated (Z-scores).

TXNIP mRNA decreases markedly in bone biopsies following treatment To screen for differentially expressed genes locally in bone tissue from CS patients, total RNA from iliac crest bone biopsies was subjected to whole-genome gene expression profiling. Of 975 significantly regulated mRNAs, TXNIP was one of the most upregulated genes in untreated vs treated CS patients $(P<0.001)$. Quantitative real-time RT-PCR validated the microarray results showing a median 1.7 -fold decrease following successful surgery $(P=0.028$; Fig. $1 \mathrm{~A})$.

Circulating TRX and biochemical bone markers in CS patients TXNIP is considered a negative regulator of TRX function and/or expression (10). We therefore analyzed serum levels of TRX in CS patients (Fig. 1B) and found significantly increased levels of TRX (median fold 1.4, $P=0.001$ ) compared with controls. Following surgical cure an additional increase was observed. A trend toward decreased serum OC was observed in CS at baseline followed by a substantial increase reaching levels markedly higher than controls after treatment (Fig. 1C). As for bone resorption, NTX was increased at baseline compared with controls and increased further following treatment (Fig. 1D).
Correlations between TXNIP, TRX and markers of bone metabolism Before treatment, the relative gene expression of TXNIP was positively correlated to circulating TRX levels $(R=0.75, n=7, P=0.05)$. Moreover, the change in TXNIP gene expression and the change in circulating TRX levels following surgical treatment tended to be correlated $(R=0.71, n=7$, $P=0.07)$. We found no correlations between changes in TXNIP gene expression in bone biopsies or changes in circulating levels of TRX to serum levels of OC or NTX (data not shown).
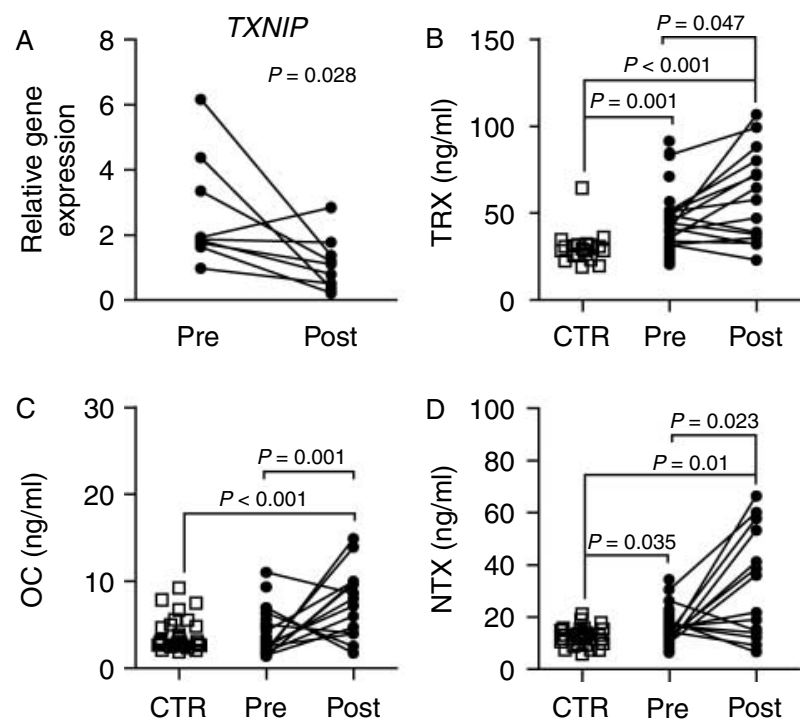

Figure 1 TXNIP gene in bone and serum measures of TRX, OC, and NTX. (A) Expression of TXNIP mRNA in bone biopsies from nine CS patients before and 3 months after surgery. Data are normalized to $\beta$-actin (Wilcoxon ranked sum test). Serum levels of (B) TRX (C) OC, and (D) NTX in 32 CS at pre-surgical levels, 16 CS at $0.9 \pm 0.9$ (mean \pm S.D.) years after surgical treatment and 29 age-, sex-, and BMI-matched controls (nonparametric Mann-Whitney between controls and CS and Wilcoxon ranked sum test between pre- and post-surgery samples). 


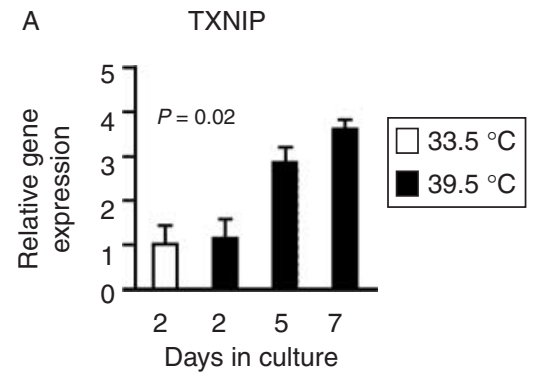

$\mathrm{D}$

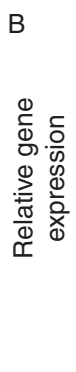

$\mathrm{E}$
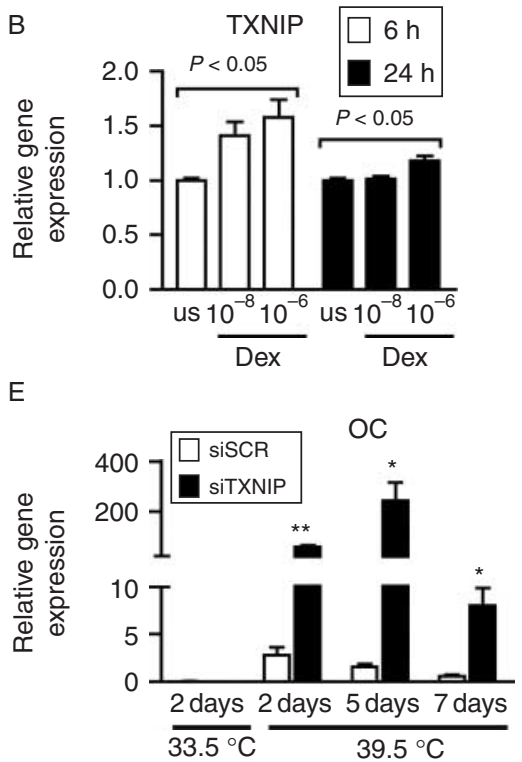

$\mathrm{H}$
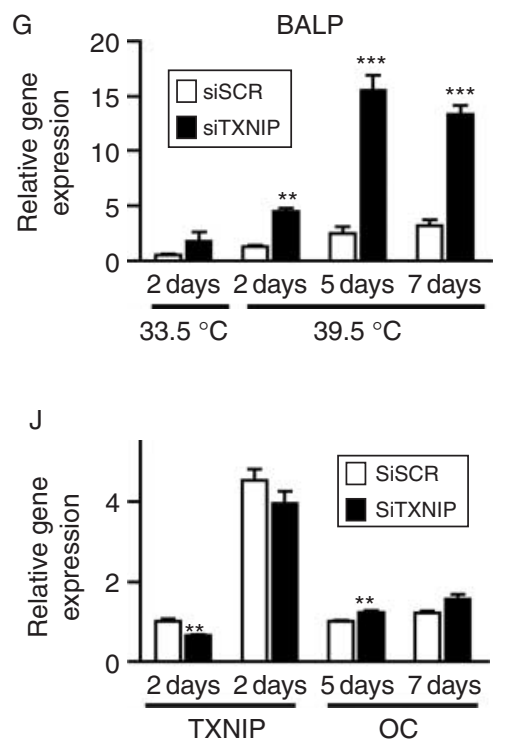

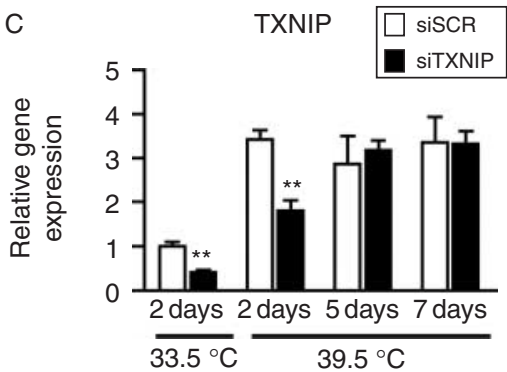

$\mathrm{F}$
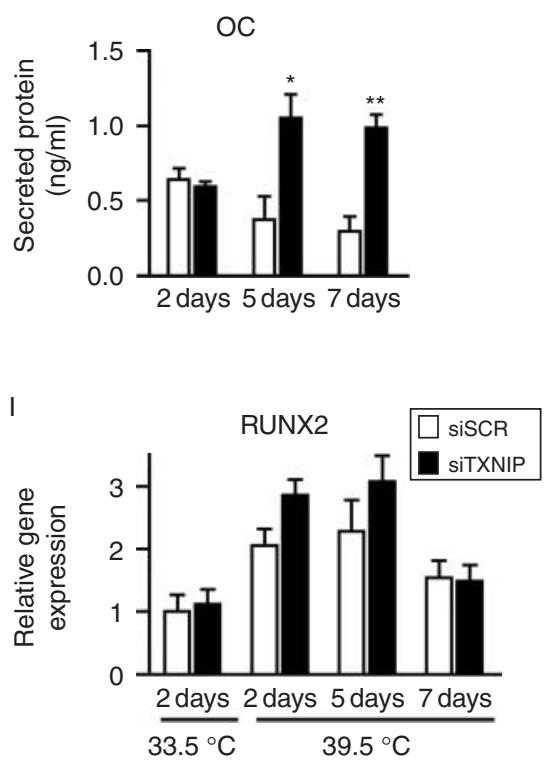

Figure 2 Increased TXNIP expression after Dex treatment and increased OC and ALP expression and protein in siTXNIP hFOB. (A) hFOB were expanded at $33.5^{\circ} \mathrm{C}$ until $\sim 90 \%$ confluent and induced to differentiate by raising the temperature to $39.5{ }^{\circ} \mathrm{C}$. At the indicated time points, expression of TXNIP mRNA was assessed by real-time RT-PCR. The data were normalized to $\beta$-actin mRNA and expressed as relative levels. $P$ values represent one-way ANOVA. (B) Cells grown to subconfluence at $33.5^{\circ} \mathrm{C}$ were induced to differentiate at $39.5^{\circ} \mathrm{C}$ for 2 days before they were treated with either vehicle (ethanol) or Dex at concentration of $10^{-6}$ and $10^{-8} \mathrm{M}$ for 6 and $24 \mathrm{~h}$. Relative mRNA levels for TXNIP were determined by real-time RT-PCR. Data are normalized to $\beta$-actin mRNA and expressed as relative levels. $P$ values represent one-way ANOVA. (C) Silencing of TXNIP in hFOB differentiated to OB. Gene expression following silencing, $24 \mathrm{~h}$ at $33.5^{\circ} \mathrm{C}+2,5$, and 7 days at $39.5^{\circ} \mathrm{C}$, using $25 \mathrm{nM}$ siTXNIP (black columns) and $25 \mathrm{nM}$ scrambled siRNA as negative control (white columns). (D) Western blot of TXNIP in total lysates from the same cultured cells. mRNAs for (D) TXNIP and (E) OC, (F) secretion of OC at 2, 5, and 7 days of cultured cells, (G) mRNAs for BALP, and (I) RUNX2. All mRNAs were normalized to $\beta$-actin and expressed as relative levels. $(\mathrm{H})$ In parallel cultures, the cells were characterized for ALP activity by histochemical staining after 5 days. (J) NHOst were silenced for 2 days, using $25 \mathrm{nM}$ siTXNIP (black columns) and $25 \mathrm{nM}$ scrambled siRNA as negative control (white columns), followed by differentiating for 2 and 7 days, measuring mRNAs for TXNIP and OC. (K) Secretion of OC at 7 days of differentiated NHOst. Results are presented as mean \pm s.E.M.; ${ }^{\star} P<0.05$, ${ }^{\star \star} P<0.01$, and ${ }^{\star \star \star} P<0.001$ indicate difference between siSCRand siTXNIP-treated cells (unpaired Student's sample $t$-test). 


\section{In vitro}

TXNIP is upregulated during hFOB differentiation and by Dex We next examined if TXNIP was regulated during differentiation or by Dex in human OB in vitro. Expression of TXNIP mRNA was assessed by real-time PCR and exhibited upregulated expression in maturing hFOB at $39.5^{\circ} \mathrm{C}$ (i.e. differentiating conditions, Fig. 2A). In hFOB cells differentiated for 2 days at $39.5^{\circ} \mathrm{C}$ and treated with two doses of Dex $\left(10^{-8}\right.$ and $\left.10^{-6} \mathrm{M}\right)$ for $6 \mathrm{~h}$ and $24 \mathrm{~h}$ respectively, TXNIP mRNA levels increased in a dose-dependent manner $(P<0.05$, Fig. $2 \mathrm{~B})$.

Silencing of TXNIP increases OB markers in hFOB and NHOst The role of TXNIP in OB development and differentiation was further assessed by silencing TXNIP mRNA expression in hFOB cells using a pool of four siRNA and scramble siRNA as a negative control. TXNIP mRNA expression was reduced by more than $50 \%$ in hFOB treated with TXNIP siRNA (siTXNIP) vs scrambled siRNA controls (siSCR) for 2 days of differentiation (Fig. 2C) and a similar and more sustained (i.e. differences also at 5 and 7 days) result was seen at protein level (Fig. 2D), resulting in significantly increased levels of typical OB-related mRNAs such as OC (Fig. 2E) and ALP (Fig. 2G) and further by ALP staining (Fig. 2H), while runt-related transcription factor 2 (RUNX2) mRNA was not affected (Fig. 2I). When investigating effects of TXNIP silencing on secreted OC, we found an increased OC secretion in the media of siTXNIP cells compared with controls (Fig. 2F). A second model of TXNIP knockdown in NHOst was used and $\sim 30 \%$ reduced TXNIP expression was obtained. Despite the low silencing result, we found a significant increase in $\mathrm{OC}$ expression and secretion from the siTXNIP NHOst after 2 and 7 days of differentiation respectively (Fig. 2J and K).

Effects of TXNIP on apoptosis in hFOB To assess the extent of plasma membrane damage, the release of the cytoplasmic enzyme LDH was quantified after 2 days of differentiation followed by $10^{-6} \mathrm{M}$ Dex treatment for $24 \mathrm{~h}$ (Fig. 3A). We found no increase of LDH release from hFOB cells treated with Dex, or between siTXNIP compared with siSCR-treated cells. In addition, the mRNAs of the BCL2/BAX ratio were not different in Dex treated or between siTXNIP- and siSCR-treated cells (Fig. 3B), but an increased expression of caspase 3 in Dex-treated cells compared with vehicle was demonstrated, and the mRNAs for caspase 3 and 8 were suppressed in siTXNIP compared with siSCR Dextreated cells (Fig. 3C and D).

Effects of TXNIP on OB-mediated osteoclastogenesis and direct effects in osteoclasts To explore if TXNIP expression in OB could affect OB-mediated osteoclastogenesis, we first investigated effects on OPG and RANKL
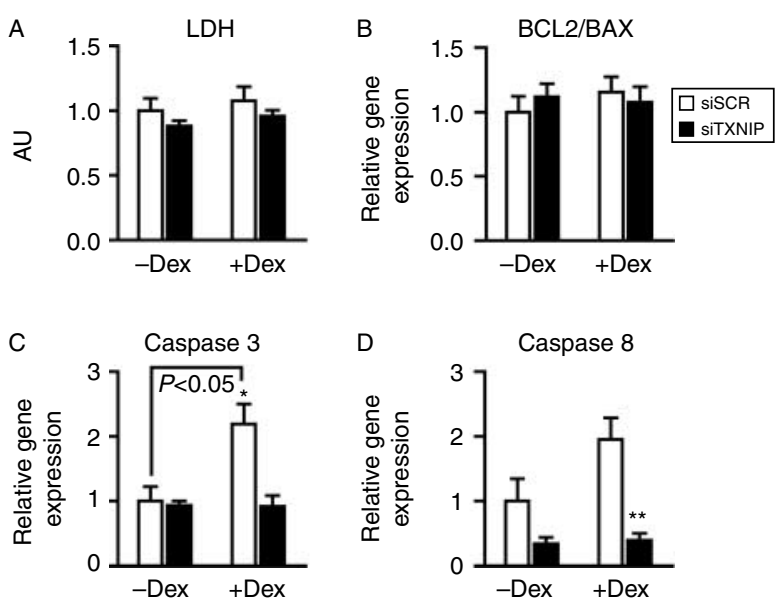

Figure 3 Decreased caspase 3 and 8 expression after silencing TXNIP in hFOB. (A) LDH cytotoxicity assay measured in supernatant of siTXNIP and siSCR following $72-\mathrm{h}$ silencing $\left(24 \mathrm{~h} 33.5^{\circ} \mathrm{C}+48 \mathrm{~h}\right.$ $39.5^{\circ} \mathrm{C}$ ) using $25 \mathrm{nM}$ siTXNIP (black columns) and $25 \mathrm{nM}$ scrambled siSCR as control (white columns), after 24-h stimulation with vehicle (US) and Dex-treated cells. Gene expression at the same time point for (B) BCL2/BAX ratio (C) caspase 3, and (D) caspase 8 were normalized to $\beta$-actin and expressed as relative mRNA levels. Results are presented as mean \pm S.E.M.; ${ }^{\star} P<0.05$ and ${ }^{* \star} P<0.01$ indicate difference between siSCR- and siTXNIP-treated cells (unpaired Student's sample $t$-test).

(TNFSF11) expression in OB. Silencing of TXNIP in hFOB increased RANKL and decreased OPG mRNA expression compared with scramble controls, leading to an increased RANKL/OPG ratio in siTXNIP-treated cells during basal conditions (Fig. 4A), thus favoring osteoclastogenesis.

To further explore TXNIP effects on OB-mediated osteoclastogenesis, human osteoclast precursor cells were treated with siTXNIP- and siSCR-conditioned media from the hFOB silencing experiment. An upregulation of tartrate-resistant acid phosphatase (TRAP) activity was detected when treating the cells with siTXNIP OB media (Fig. 4B). To explore TXNIP function on bone resorption in a bone resorption model, we used human bone plates coated with osteoclasts differentiated from CD14 + cells. Using an siTXNIP OB medium, an increased calcium release from the bone was detected, indicating higher resorption activity with the siTXNIP OB medium (Fig. 4C). Differentiation of human osteoclast precursors showed no regulation of TXNIP expression (Fig. 3D). However, treating the cells with different doses of Dex showed an increased TXNIP expression (Fig. 3E). To explore direct effects on TXNIP in osteoclasts, we silenced TXNIP and found no difference of TRAP activity in the condition media in siTXNIPtreated osteoclasts compared with siSCR-treated osteoclasts (Fig. 4F). All osteoclast experiments were validated in osteoclasts differentiated from $\mathrm{CD} 14+$ monocytes (data not shown). The main findings of the present study are summarized in Fig. 5. 


\section{Discussion}

In the present study, we show that TXNIP expression in bone biopsies from CS patients is highly downregulated following surgical treatment. Circulating levels of TRX were increased in the patients at baseline and increased further in relation to treatment. Furthermore, we show that TXNIP is upregulated during OB differentiation in vitro and by Dex stimulation of $\mathrm{OB}$ in a dosedependent manner. Finally, our study indicates that TXNIP may regulate osteoclastogenesis through OB signaling. These data suggest that TXNIP is highly upregulated by GC in bone tissue and that TXNIP by itself is of importance for bone metabolism.

We performed global gene expression profiling of bone biopsies from CS patients before and after treatment and found that the gene TXNIP is highly expressed and significantly downregulated following successful surgery. These clinical translational findings are in accordance with previous studies showing TXNIP to be induced by GC in a murine T-cell lymphoma cell line (11). However, bone biopsies consist of different cell types (e.g. OB, osteoclasts, osteocytes, and mesenchymal stem cells) and our data reflect the overall tissue levels of transcripts, and not their precise cellular source. TXNIP gene expression at baseline was associated with increased circulating level of TRX also at baseline and following treatment, substantiating the close relation between the two proteins $(9,10)$. In accordance with the decrease in TXNIP in bone biopsies following surgical cure and normalization of cortisol levels, we found TXNIP to be upregulated in a dose-dependent manner by Dex in human OB and osteoclasts in vitro. Moreover, TXNIP was also upregulated during $\mathrm{OB}$ differentiation. Furthermore, silencing of TXNIP gene expression in OB showed a sustained increase in levels of typical OB markers (e.g. OC and bone specific alkaline phosphatase (BALP)). These findings, indicating a considerable attenuating effect of TXNIP on OB differentiation and function, are in agreement with the bone formative phenotype observed in active CS,

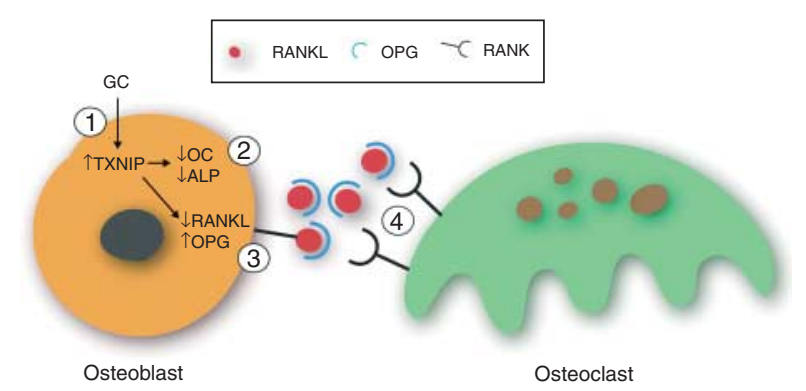

Figure 5 Model of potential effects of TXNIP regulation in bone cells. (1) GC may increase TXNIP in OB. (2) Increased TXNIP decreases differentiation of $O B$ and expression of OC and ALP. (3) Increased TXNIP increases OPG and decreases RANKL leading to (4) decreased OB-mediated osteoclastogenesis in uncoupling of bone turnover. 
characterized by decreased OB number and function with low levels of biochemical markers of bone formation (3), as well as the rapid increase in bone turnover with increased bone formation, following normalization of cortisol levels. GCs promote the apoptosis of $\mathrm{OB}$ and osteocytes in vitro via the activation of caspases $(1,19)$. We found no evidence of decreased apoptosis in silenced TXNIP hFOB, but an increased expression after Dex treatment in caspase 3 and a suppressed expression after silencing TXNIP of caspase 3 and 8 were observed, and supports the possible suppressive effects of TXNIP on OB. GC also represses transcription of OC through a GC response element (20), and thereby making the interpretation of GC effects on OC changes in CS complicated. We found OC to be upregulated by silencing TXNIP, without Dex in the experiment, thus suggesting TXNIP to be directly involved in OC suppression. In this study, the cellular effects were searched on the hFOB cell line. From the review of Liu et al. (21), some important issues about different $\mathrm{OB}$ models in vitro have been addressed. The most widely used $\mathrm{OB}$ model systems in vitro are primary cultures derived from normal human and rodent bone fragments, or osteosarcoma cell lines generated from human bone tumors. Each of these cell sources has strengths and limitations. In effort to overcome these limitations, Harris et al. (16) established a conditionally immortalized, human-fetal-OB cell line, hFOB 1.19 that was stably transfected with a gene coding for a temperature-sensitive mutant of the SV40 large T antigen. Resultant hFOB cells express OB-specific phenotypic markers and mineralize extracellular matrix. Later, Subramaniam et al. (22) characterized hFOB 1.19 as an immortalized, but nontransformed, cell line with minimal chromosome abnormalities and normal spectrum of matrix proteins. They reported that the hFOB cells appeared to be an excellent model system for the study of OB biology in vitro. In a previous study (12) we assessed transcripts characteristically expressed by OB; i.e. ALP, $\mathrm{OC}$, and COL1A2, and the bone-remodeling cytokines OPG and RANKL in relation to differentiation and Dex treatment. We demonstrated satisfying results for a normal OB phenotype. Another important advantage of this cell line is property that the cells are fast differentiated into mature $\mathrm{OB}$, compared with normal $\mathrm{OB}$, which need $\sim 3$ weeks for full differentiation. The hFOB are also easy to transfect and therefore an excellent model to study mechanisms with RNA interference. Another limitation in the present study is the lack of bone biopsies from a control group for comparing TXNIP expression in bone in vivo. However, although we cannot establish if the TXNIP in bone is increased in active CS, the strong correlation between TXNIP and TRX, as well as the markedly elevated TRX levels compared with control at baseline, may suggest so.

The OPG/RANKL system is of major importance for the coordinated coupling principle in bone remodeling $(4,23)$. We found that silencing of TXNIP in hFOB decreased OPG and increased RANKL, indicating that decreased OB expression of TXNIP favors bone resorption.
Indeed, when mature osteoclasts were cultured with conditioned media from siTXNIP OB, an increase in TRAP activity and calcium release from bone in a bone resorption model was observed. Although these data, indicating a limiting effect of increased TXNIP on OB-mediated bone resorption, may seem in contrast with the slightly enhanced bone resorption observed in CS patients, they possibly indicate that in the presence of excess cortisol the effect of TXNIP on bone resorption is limited, and that direct effects of GC on osteoclasts dominate. However, following surgical cure, a marked increase in bone turnover, with increased bone formation and resorption, is observed. Thus, the decline in TXNIP following surgery and normalization of cortisol levels, leading to enhanced osteoblastogenesis, may favor bone resorption through an increased RANKL/OPG ratio, and thereby explaining our previous observation of a restoration of coupling of bone remodeling following the treatment of CS (14). Together, these observations imply that TXNIP may be a major regulator of OB-mediated osteoclastogenesis.

In conclusion, the present translational study showed that TXNIP expression is highly upregulated in bone tissue during treatment of active CS, and by GC in bone cells in vitro. Moreover, our data indicate that TXNIP may mediate some of the detrimental effects of GC on $\mathrm{OB}$ function as well as modulate OB-mediated osteoclastogenesis by regulating the OPG/RANKL ratio, possibly playing a role in the pathogenesis of human glucocorticoidinduced osteoporosis.

\section{Declaration of interest}

The authors declare that there is no conflict of interest that could be perceived as prejudicing the impartiality of the research reported.

\section{Funding}

This research did not receive any specific grant from any funding agency in the public, commercial or not-for-profit sector.

\section{Acknowledgements}

The authors are grateful to Dr Ole Kristoffer Olstad and the Affymetrix Microarray Core Facility at Oslo University Hospital/Ullevål for performing and analyzing the microarray experiment.

\section{References}

1 Bouvard B, Legrand E, Audran M \& Chappard D. Glucocorticoidinduced osteoporosis: a review. Clinical Reviews in Bone and Mineral Metabolism 20108 15-26. (doi:10.1007/s12018-009-9051-9)

2 Kaltsas G \& Makras P. Skeletal diseases in Cushing's syndrome: osteoporosis versus arthropathy. Neuroendocrinology 201092 (Suppl 1) 60-64. (doi:10.1159/000314298)

3 Hofbauer LC, Gori F, Riggs BL, Lacey DL, Dunstan CR, Spelsberg TC \& Khosla S. Stimulation of osteoprotegerin ligand and inhibition of osteoprotegerin production by glucocorticoids in human osteoblastic lineage cells: potential paracrine mechanisms of glucocorticoid-induced osteoporosis. Endocrinology $1999 \mathbf{1 4 0}$ 4382-4389. (doi:10.1210/en.140.10.4382) 
4 Lacey DL, Timms E, Tan HL, Kelley MJ, Dunstan CR, Burgess T, Elliott R, Colombero A, Elliott G, Scully S, Hsu H, Sullivan J, Hawkins N, Davy E, Capparelli C, Eli A, Qian YX, Kaufman S, Sarosi I, Shalhoub V, Senaldi G, Guo J, Delaney J \& Boyle WJ. Osteoprotegerin ligand is a cytokine that regulates osteoclast differentiation and activation. Cell $1998 \quad 93$ 165-176. (doi:10.1016/S0092-8674(00)81569-X)

5 Kakisaka Y, Nakashima T, Sumida Y, Yoh T, Nakamura H, Yodoi J \& Senmaru H. Elevation of serum thioredoxin levels in patients with type 2 diabetes. Hormone and Metabolic Research 200234 160-164. (doi:10.1055/s-2002-23201)

6 Miyamoto S, Kawano H, Hokamaki J, Soejima H, Kojima S, Kudoh T, Nagayoshi Y, Sugiyama S, Sakamoto T, Yoshimura M, Nakamura H, Yodoi J \& Ogawa H. Increased plasma levels of thioredoxin in patients with glucose intolerance. Internal Medicine 200544 1127-1132. (doi:10.2169/internalmedicine.44.1127)

7 Okuda M, Inoue N, Azumi H, Seno T, Sumi Y, Hirata K, Kawashima S, Hayashi Y, Itoh H, Yodoi J \& Yokoyama M. Expression of glutaredoxin in human coronary arteries: its potential role in antioxidant protection against atherosclerosis. Arteriosclerosis, Thrombosis, and Vascular Biology 200121 1483-1487. (doi:10.1161/hq0901.095550)

8 Kaimul AM, Nakamura H, Masutani H \& Yodoi J. Thioredoxin and thioredoxin-binding protein-2 in cancer and metabolic syndrome. Free Radical Biology E Medicine 200743 861-868. (doi:10.1016/ j.freeradbiomed.2007.05.032)

9 Kim SY, Suh HW, Chung JW, Yoon SR \& Choi I. Diverse functions of VDUP1 in cell proliferation, differentiation, and diseases. Cellular \& Molecular Immunology 20074 345-351.

10 Nishiyama A, Matsui M, Iwata S, Hirota K, Masutani H, Nakamura H, Takagi Y, Sono H, Gon Y \& Yodoi J. Identification of thioredoxin-binding protein-2/vitamin $\mathrm{D}(3)$ up-regulated protein 1 as a negative regulator of thioredoxin function and expression. Journal of Biological Chemistry $199927421645-$ 21650. (doi:10.1074/jbc.274.31.21645)

11 Wang Z, Rong YP, Malone MH, Davis MC, Zhong F \& Distelhorst CW. Thioredoxin-interacting protein (txnip) is a glucocorticoid-regulated primary response gene involved in mediating glucocorticoid-induced apoptosis. Oncogene 200625 1903-1913. (doi:10.1038/sj.onc.1209218)

12 Lekva T, Bollerslev J, Kristo C, Olstad OK, Ueland T \& Jemtland R. The glucocorticoid-induced leucine zipper gene (GILZ) expression decreases after successful treatment of patients with endogenous Cushing's syndrome and may play a role in glucocorticoidinduced osteoporosis. Journal of Clinical Endocrinology and Metabolism 201095 246-255. (doi:10.1210/jc.2009-0595)

13 Godang K, Ueland T \& Bollerslev J. Decreased bone area, bone mineral content, formative markers, and increased bone resorptive markers in endogenous Cushing's syndrome. European Journal of Endocrinology 1999141 126-131. (doi:10.1530/eje.0.1410126)

14 Kristo C, Jemtland R, Ueland T, Godang K \& Bollerslev J. Restoration of the coupling process and normalization of bone mass following successful treatment of endogenous Cushing's syndrome: a prospective, long-term study. European Journal of Endocrinology 2006154 109-118. (doi:10.1530/eje.1.02067)

15 Bollerslev J, Steiniche T, Melsen F \& Mosekilde L. Structural and histomorphometric studies of iliac crest trabecular and cortical bone in autosomal dominant osteopetrosis: a study of two radiological types. Bone 198910 19-24. (doi:10.1016/87563282(89)90142-7)

16 Harris SA, Enger RJ, Riggs BL \& Spelsberg TC. Development and characterization of a conditionally immortalized human fetal osteoblastic cell line. Journal of Bone and Mineral Research 199510 178-186. (doi:10.1002/jbmr.5650100203)

17 Hicok KC, Thomas T, Gori F, Rickard DJ, Spelsberg TC \& Riggs BL. Development and characterization of conditionally immortalized osteoblast precursor cell lines from human bone marrow stroma. Journal of Bone and Mineral Research 199813 205-217. (doi:10.1359/jbmr.1998.13.2.205)

18 Nissen-Meyer LS, Jemtland R, Gautvik VT, Pedersen ME, Paro R, Fortunati D, Pierroz DD, Stadelmann VA, Reppe S, Reinholt FP, Del Fattore A, Rucci N, Teti A, Ferrari S \& Gautvik KM. Osteopenia, decreased bone formation and impaired osteoblast development in Sox4 heterozygous mice. Journal of Cell Science $2007 \mathbf{1 2 0}$ 2785-2795. (doi:10.1242/jcs.003855)

19 Chua CC, Chua BH, Chen Z, Landy C \& Hamdy RC. Dexamethasone induces caspase activation in murine osteoblastic MC3T3-E1 cells. Biochimica et Biophysica Acta 20031642 79-85. (doi:10.1016/S0167-4889(03)00100-9)

20 Morrison NA, Shine J, Fragonas JC, Verkest V, McMenemy ML \& Eisman JA. 1,25-Dihydroxyvitamin D-responsive element and glucocorticoid repression in the osteocalcin gene. Science 1989 246 1158-1161. (doi:10.1126/science.2588000)

21 Liu X, Lim JY, Donahue HJ, Dhurjati R, Mastro AM \& Vogler EA. Influence of substratum surface chemistry/energy and topography on the human fetal osteoblastic cell line hFOB 1.19: phenotypic and genotypic responses observed in vitro. Biomaterials $2007 \mathbf{2 8}$ 4535-4550. (doi:10.1016/j.biomaterials.2007.06.016)

22 Subramaniam M, Jalal SM, Rickard DJ, Harris SA, Bolander ME \& Spelsberg TC. Further characterization of human fetal osteoblastic hFOB 1.19 and hFOB/ER alpha cells: bone formation in vivo and karyotype analysis using multicolor fluorescent in situ hybridization. Journal of Cellular Biochemistry 200287 9-15. (doi:10.1002/jcb.10259)

23 Camozzi V, Sanguin F, Albigier N, Scaroni C, Mantero F, Zaninotto M, Frigo A, Piccolo M \& Luisetto G. Persistent increase of osteoprotegerin levels after cortisol normalization in patients with Cushing's syndrome. European Journal of Endocrinology 2010 162 85-90. (doi:10.1530/EJE-09-0800)

Received 19 December 2011

Revised version received 21 March 2012

Accepted 26 March 2012 